\title{
Herramientas de cribado para la detección de retrasos o trastornos en el desarrollo: Una revisión sistemática de la literatura
}

\section{Screening tools for detection of delays or developmental disorders: a systematic review of the literature}

\begin{abstract}
Resumen
El objetivo del artículo es analizar

los diferentes instrumentos de

detección de trastornos o retraso en el desarrollo para valorar la calidad y aplicabilidad de los mismos. Se realiza una revisión sistemática de las publicaciones científicas publicadas en los últimos 25 años (I990-20I4) e indexadas en bases de datos internacionales disponibles en línea. El estudio se limita a herramientas de detección basadas en cuestionarios, tests o sistemas de observación de la conducta del niño. Las herramientas o sistemas analizados son valorados según una escala de calidad construida "ad hoc". Los resultados demuestran una gran variabilidad de herramientas y sistemas de detección, muchos de ellos son cuestionarios de investigación, otros son herramientas de diagnóstico utilizadas con fines de detección. Se pueden clasificar en dos grandes bloques, los construidos en base a los hitos evolutivos cuyo incumplimiento temporal puede significar un retraso en el desarrollo y aquellos otros que, asumiendo el primer principio han seleccionado los hitos que pueden ser precursores de trastornos del desarrollo.

En las conclusiones se realizan recomendaciones para la validación de los sistemas de detección en España y la construcción de nuevos.
\end{abstract}

\section{Palabras clave}

Revisión sistemática, detección, trastornos del desarrollo, retraso en el desarrollo.

\begin{abstract}
The goal of this article is to analyze the quality and applicability screening instruments aimed at detecting developmental delays or disorders. A systematic review of the scientific literature published during the last 25 years (I990-20I4) retrieved from different databases is carried out. The study is limited to detection systems based on questionnaires, tests or observation of children`s behavior. The analyzed systems are rated on a scale of quality built "ad hoc". The results show a high variability of tools and detection systems, many of which are research questionnaires, while others are diagnostic tools used for screening purposes. They can be classified in two large groups of instruments: I), the ones based on developmental milestones whose temporary failure can mean a delay in the development and 2), those who, assuming the first principle, have identified the milestones that can be precursors of developmental disorders. In the conclusions, recommendations are offered regarding the validation of screening systems in Spain and for the construction of new ones.
\end{abstract}

\section{Keywords}

Systematic review, screening, developmental disorders, developmental delay.

\section{Francisco Alcantud Marín <francisco.alcantud@uv.es>}

Universitat de València, Catedrático de Psicología Evolutiva y de la Educación. Director del Centro Universitario de Diagnóstico y Atención Temprana de la Universitat de València

\section{Yurena Alonso Esteban \\ <yurena.alonso@uv.es>}

Departamento de Psicología Evolutiva y de la Educación de la Universitat de Valencia

\section{Darío Rico Bañón \\ <dario.rico@uv.es>}

Centro Universitario de Diagnóstico y Atención Temprana de la Universitat de Valencia

Para citar:

Alcantud Marín, F. et al. (2015):

"Herramientas de cribado para la detección de retrasos o trastornos en el desarrollo: Una revisión sistemática de la literatura", Revista Española de Discapacidad, 3 (2): 7-26.

Doi: <http://dx.doi.org/IO.5569/23405 IO4.03.02.0I>

Fecha de recepción: 29-09-20I 5 Fecha de aceptación: 09-I2-20I 5 


\section{Declaración de conflicto de intereses}

Los autores afirman no tener conflicto de intereses aun siendo los autores del Sistema de Detección Precoz de Trastornos del Desarrollo (SDPTD), puesto que el contenido de este artículo (aunque en la versión publicada este actualizado hasta diciembre del 20I4), fue desarrollado antes del desarrollo del SDPTD y como fase previa del proyecto de investigación. Aun así, para evitar conflictos, se ha excluido expresamente del análisis el sistema del que ellos son autores.

\section{Introducción}

Desde hace ya tiempo se ha puesto de manifiesto la necesidad de desarrollar sistemas de cribado del desarrollo infantil (Shonkoff et al. I979). Los llamados "cribados del desarrollo infantil" son sistemas desarrollados con el objetivo de detectar algún tipo de retraso o trastorno en el desarrollo infantil (motor, cognitivo, socio-emocional, etc.). Se trata en general de cuestionarios o listas de chequeo que facilitan la observación y seguimiento del desarrollo por el profesional. Otros son listados de síntomas o conductas que han manifestado evidencias de relacionarse con trastornos diagnosticados con posterioridad. Los resultados de su uso han demostrado, no solo que los sistemas de cribado del desarrollo son una medida preventiva (Hix-Small et al. 2006), sino que si se acompañan de programas de atención temprana aplicados de forma precoz, producen beneficios en la familia y en el niño/a a largo plazo. La rentabilidad social y económica es incuestionable (Arruabarrena y de Paul, 20I2; Rydz et al. 2006).

Según la Sociedad de Neurología Infantil (Filipek et al. 2000), el Instituto de Salud Carlos III (Muñoz Yunta et al. 2006) y otras instituciones e investigadores, las herramientas de cribado pueden clasificarse en tres niveles:
- $\quad$ Nivel I: Corresponde a los sistemas de vigilancia del desarrollo, por tanto, de aplicación universal. Se suelen aplicar en las consultas de pediatría o en las aulas del primer ciclo de educación infantil (o-3 años). Su objetivo es detectar si el niño/a evaluado/a presenta riesgo de algún trastorno del desarrollo. El síntoma buscado es el retraso en la adquisición de determinados hitos evolutivos que se consideran clave en el desarrollo del niño/a. La herramienta de cribado de primer nivel más frecuente, suele ser el juicio diagnóstico del profesional apoyado de algún tipo de lista de chequeo. Estas listas de chequeo pueden ser administradas por los propios profesionales o contestadas por los padres/madres o cuidadores del niño/a y contrastadas posteriormente por los/as profesionales.

- Nivel II: Corresponde a instrumentos que tienen como objetivo identificar sobre los/ as niños/as de la población detectada con riesgo de desarrollo atípico en el nivel I, si específicamente tienen riesgo de padecer un trastorno o una patología concreta. Las herramientas de cribado de segundo nivel pueden confundirse con las herramientas de diagnóstico (Coonrod y Stone, 2005; Lord y Corsello, 2005). Normalmente requieren mayor tiempo y entrenamiento que las de primer nivel y pueden ser utilizadas como parte de la evaluación inicial en el proceso diagnóstico aunque no como herramienta única del mismo (Johnson y Myers, 2007).

- $\quad$ Nivel III: Corresponde a los instrumentos utilizados para la confirmación diagnóstica. Suelen ser instrumentos específicos que evalúan una o varias áreas o subáreas del desarrollo. Su administración es realizada por profesionales con entrenamiento específico en cada uno de los instrumentos utilizados. Existen escalas del desarrollo con formas breves, a modo de screening, como la Bayley (Bayley, 2006) o la Battelle (Newborg, 2005). Estas formas breves no se suelen utilizar como sistemas de detección de nivel I aunque su objetivo sea la detección del retraso en el desarrollo como síntoma de un posible trastorno. 
Figura 1. Niveles de detección

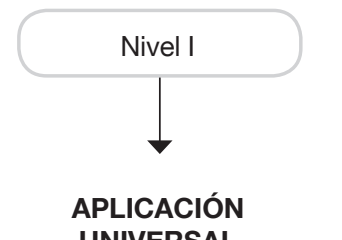

UNIVERSAL

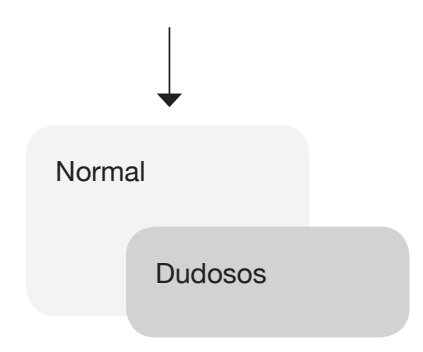

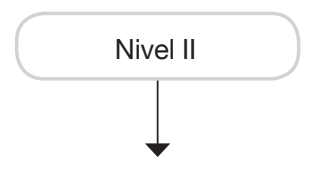

APLICACIÓN POBLACIÓN DE RIESGO

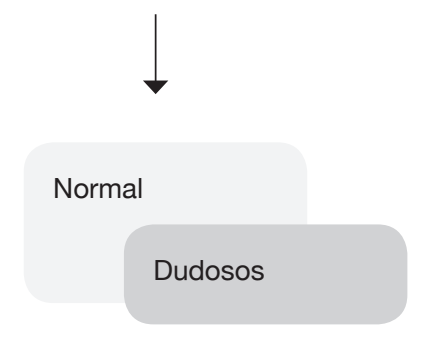

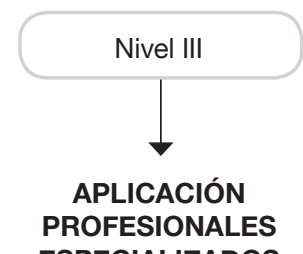

ESPECIALIZADOS

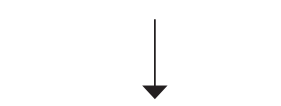

Normal

Patológicos

Fuente: Elaboración propia.

El diagnóstico confiable de los trastornos del desarrollo de etiología idiopática no se realiza hasta los 24-36 meses de vida o incluso, según los casos, posteriormente. Otros, por el contrario, conocida su etiología y sus marcadores biológicos, pueden ser diagnosticados en el mismo momento del nacimiento. Por ejemplo, en el caso de los TEA el diagnóstico puede establecerse sobre los 20-24 meses (Cox et al. I999; Dawson et al. 2000; Bryson et al. 2008; Kleinman et al. 2008). Otros trastornos del desarrollo como el déficit cognitivo o el retraso mental serán mucho más tardíos al requerir una evaluación formal de las competencias cognitivas mediante una prueba psicométrica de inteligencia. La detección precoz permite, aunque no se tenga un diagnóstico formal, la intervención temprana (GAT, 2000). Por este motivo el suelo de un sistema de detección debe ser muy bajo en edad. Por otra parte, es conocido que el diagnóstico ya es posible en algunos casos sobre los 36-48 meses de vida, este debería ser el techo puesto a esa edad. Ante la sospecha, se puede aplicar instrumentos diagnósticos directamente sin necesidad de utilizar previamente instrumentos de detección. Por otra parte, el incremento de la prevalencia de algunos trastornos del neurodesarrollo y la evidencia de la eficacia de los programas de intervención temprana cuando se inician antes de los dos años, ha generado que instrumentos de detección específicos de nivel II se recomienden aplicar de forma universal, es decir, como si se tratara de nivel I. Esta forma de uso ha hecho que se incremente la confusión sobre cuál es el sentido de los niveles de detección.

Éste artículo se centra en el estudio de las herramientas de detección de los trastornos o retrasos del desarrollo. El término "retraso en el desarrollo" es utilizado en muchas ocasiones para identificar a los/as niños/as que no alcanzan los hitos evolutivos en el momento en que lo alcanza el promedio de la población de su edad. Este hecho, tradicionalmente se ha considerado como una señal de alerta de un posible trastorno del desarrollo. Sin embargo, en la literatura científica no existe un consenso sobre el significado del término ni de cuando utilizarlo (Kube et al. ı988). El significado más común ha sido el de retraso mental (trastorno del desarrollo intelectual). También se utiliza en ocasiones para referirse a 
los/as niños/as que obtienen puntuaciones bajas en las escalas de desarrollo o para identificar a los/as niños/as nacidos pretérmino sanos que obtienen puntuaciones más bajas en las escalas de desarrollo que sus iguales nacidos a término (dentro de un desarrollo normativo). Se ha utilizado también como equivalente al término "retraso psicomotor" (Fenichel, I988). En general, es aceptado como un diagnóstico provisional, utilizado hasta que el niño/a tiene la edad suficiente para ser evaluado con pruebas psicométricas formales. En general, el retraso en el desarrollo es un concepto que está siendo reemplazado por el de "trastornos en el desarrollo”. La razón fundamental es que el primero de ellos traslada la idea de poca gravedad y en ocasiones induce a la espera en la intervención. Hoy en día podría referirse a ellos como "trastornos del neurodesarrollo". Es decir, aquellas alteraciones en el desarrollo de funciones vinculadas a la maduración del sistema nervioso central que se inician antes de que finalice el proceso de maduración del mismo y siguen un curso evolutivo estable (ArtigasPallarés, 20I I). Sin embargo, cuando sólo se tienen evidencias de un retraso en el desarrollo sin conocer las causas, y sin tener un diagnóstico específico, se suele mantener el término genérico de "trastornos del desarrollo".

En este artículo se revisa la literatura científica sobre el uso y desarrollo de herramientas de cribado o detección de los trastornos o retrasos del desarrollo de nivel I, es decir, de posible aplicación universal con el objetivo de valorar la calidad de los sistemas de detección, su aplicabilidad y las características que los hacen más adecuados. Para ello, se realiza una revisión sistemática de las publicaciones indexadas en las bases de datos científicas afines a las áreas de conocimiento con competencia (medicina, psicología, educación). El objetivo de esta revisión es detectar todas las posibles herramientas de detección de aplicación universal (nivel I) de trastornos o retrasos en el desarrollo infantil con la finalidad de valorar su calidad analizando sus fortalezas y debilidades. Esta revisión se realiza en el marco de una investigación de mayor nivel que tuvo como objetivo la construcción de un nuevo sistema de detección de los trastornos del desarrollo (Alcantud et al. 2015). Los resultados de revisión que se presentan en esta publicación han sido actualizados hasta diciembre del 2014.

\section{Método y procedimiento}

Se ha desarrollado una revisión de la literatura utilizando principios sistemáticos de búsqueda y selección ${ }^{\mathrm{I}}$. El objetivo es seleccionar artículos de revistas científicas (que incorporen evaluación por pares, sin criterio de tiempo) que permitan identificar las herramientas de cribado para detectar niños/as menores de tres años con riesgo de presentar trastornos del desarrollo. Para ello se realizó la búsqueda utilizando en primer lugar la base de datos en línea Proquest Psychology Journals-PPJ, accediendo mediante la plataforma TROBES del Servicio de Bibliotecas y Documentación de la Universitat de València. En principio se ensayaron búsquedas con diferentes palabras como "Early detection", "Screening tools", etc., determinando que las palabras o frases que más se ajustaban al objetivo de búsqueda eran "Developmental Screening test" o "Developmental Screening Tools" y que podrían aparecen en cualquier campo de la ficha del documento indexado (incluyendo los resúmenes o “abstracts” en inglés para los documentos escritos en otro idioma). El trabajo más antiguo detectado es del año I975 (Barnes y Stark, I975) y se trata de la publicación del estudio normativo del test de screening de Denver. Dado el gran volumen de información obtenida, se optó por limitar el periodo de búsqueda a los documentos publicados en los últimos 25 años (I990-20I4), encontrándose en total 250 artículos. Una vez realizada la búsqueda en esta primera base de datos, se extendió a otras bases de datos en línea de la misma plataforma que tuvieran actualizaciones permanentes (mínimo

I. NHS Centre for Reviews and Dissemination: Systematic reviews. CRD's guidance for undertaking reviews in health care University of York; 2008 [http://www.york.ac.uk/inst/crd/ pdf/Systematic_Reviews.pdf]. 
mensual) y dado el carácter multidisciplinar de la temática de estudio, consideradas de interés por cubrir áreas de conocimiento afines. Las bases de datos consultadas fueron: Medicina+ (PubMed MEDLINE), Educación (ProQuest ERIC) y test psicológicos en general (ProQuest PsycTESTC).

\subsection{Criterios de Inclusión-Exclusión}

Aunque la selección de las bases de datos se realizó cubriendo diferentes áreas de conocimiento como la psicología, educación y medicina, se detectaron un gran número de artículos duplicados. Todos los artículos fueron copiados sobre RefWorks, desde donde se realizó la eliminación de los duplicados y un primer análisis cualitativo de los artículos preseleccionados mediante la lectura de los resúmenes, verificando el cumplimiento de los criterios de inclusión (estudios psicométricos de herramientas de detección de trastornos del desarrollo de nivel I, aplicaciones comunitarias de las mismas, adaptaciones transculturales y lingüísticas, etc.). Fueron excluidos aquellos que cumplían alguno de los siguientes criterios de exclusión: estudios de prevalencia, estudios en poblaciones superiores a tres años, estudios de intervención, técnicas no psicométricas u observacionales de detección, estudios de

\section{Figura 2. Flujo del proceso de búsqueda documental}

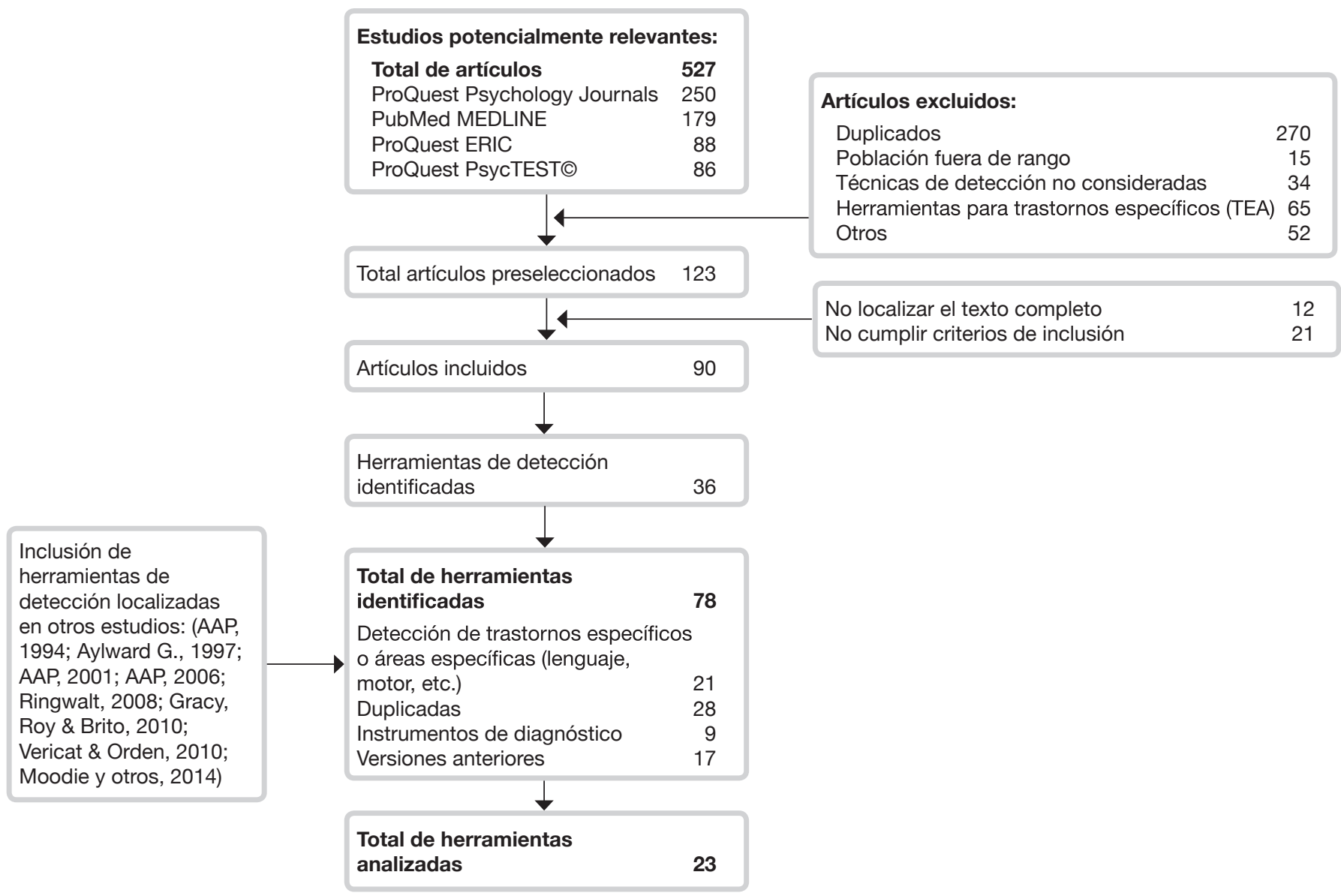

Fuente: Elaboración propia. 
biomarcadores, herramientas de diagnóstico, cribados específicos en áreas concretas de desarrollo (motor, comunicación y lenguaje, desarrollo socio-emocional) o sobre patologías concretas (TEA, TDAH, etc.). Del total de artículos se pre-seleccionaron por cumplir los criterios de búsqueda un total de $\mathbf{2} 3$ artículos.

Se aplicó un segundo análisis cualitativo de los artículos mediante la lectura en su versión completa. Ambos análisis fueron realizados inicialmente por una persona y chequeados por un segundo juez. En este proceso se exclouyeron I 2 artículos por no ser accesible el texto completo y 2 I por incumplir alguno de los criterios de inclusión-exclusión. En total se analizan los 90 artículos incluidos en la revisión. De este conjunto de artículos se detectan un total de 36 herramientas o sistemas de detección.

Entre todos los artículos se detectaron también estudios de revisión previos de los que se vaciaron todas las herramientas de detección (Aylward, I997; AAP, I994; AAP, 200Ia; AAP, 200Ib; AAP, 2006; Moodie et al. 20I4; Gracy et al. 2010; Ringwalt, 2008; Bedford et al. 2013). Entre estas revisiones destacamos la de Vericat y Orden (2010) dado que aporta información de pruebas desarrolladas en español, sin embargo, no han sido incluidas la mayoría de ellas al no estar accesible o ser ilocalizable la información psicométrica de las mismas.

Se listaron todas las herramientas de detección referenciadas en todos los documentos eliminándose las duplicadas, las versiones obsoletas, las herramientas de diagnóstico utilizadas como screening o las herramientas de detección específicas de un tipo de trastorno o un área de desarrollo en particular (lenguaje, desarrollo emocional, motor, etc.), quedando definitivamente el listado formado por 23 herramientas o sistemas de detección.

A continuación, la búsqueda se amplió utilizando los nombres de las herramientas de detección como elemento de búsqueda en "Proquest Psychology Journals". Se organizó la información de todos los artículos donde se referenciaba el uso o estudio de cada una de las diferentes herramientas según el tipo de estudio: adaptaciones culturales o traducciones a otras lenguas, validación con otros instrumentos de cribado, estudios psicométricos, aplicaciones comunitarias, etc. Estas herramientas se presentan en la tabla I donde se incluye una breve descripción de las mismas y las propiedades psicométricas más relevantes.

\begin{tabular}{|c|c|c|c|c|c|c|}
\hline \multirow[b]{2}{*}{ Nombre } & \multirow[b]{2}{*}{ Autor/es } & \multirow[b]{2}{*}{ Descripción: } & \multicolumn{2}{|c|}{ Edad } & \multirow{2}{*}{ 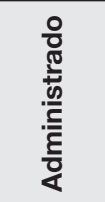 } & \multirow{2}{*}{ 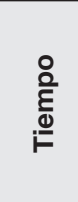 } \\
\hline & & & $\begin{array}{l}\frac{\circ}{0} \\
\stackrel{\vec{D}}{0}\end{array}$ & 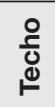 & & \\
\hline $\begin{array}{l}\text { 1.- Ages } \\
\text { and Stages } \\
\text { Questionnaires } \\
\text { (ASQ-3). }\end{array}$ & $\begin{array}{l}\text { (Squires et } \\
\text { al.1997; } \\
\text { Squires et al. } \\
\text { 1998). }\end{array}$ & $\begin{array}{l}\text { Existen diferentes versiones, la más actual (ASQ- } \\
\text { 3), está integrada por } 21 \text { cuestionarios. Está } \\
\text { diseñado para ser contestado por los/as padres/ } \\
\text { madres y posteriormente puntuados por los/ } \\
\text { as profesionales. Evalúa diferentes áreas del } \\
\text { desarrollo (lenguaje, socio-emocional, motricidad } \\
\text { y cognición) en cada edad. Existen versiones } \\
\text { para respuesta cara a cara, por correo y "online". }\end{array}$ & 1 & 66 & Padres & $10-15$ \\
\hline $\begin{array}{l}\text { 2.- Battelle } \\
\text { Developmental } \\
\text { Inventory } \\
\text { Screening Test, } \\
2^{\text {nd }} \text { ed.(BDI-ST). }\end{array}$ & $\begin{array}{l}\text { (Newborg, } \\
2005 \text { ). }\end{array}$ & $\begin{array}{l}\text { Consiste en } 100 \text { ítems ( } 20 \text { para cada dominio o } \\
\text { área evaluada) en diez niveles de edad. Evalúa } \\
\text { las áreas de lenguaje expresivo y receptivo, } \\
\text { motora gruesa y fina, adaptativa, personal-social } \\
\text { y área cognitiva/académica. }\end{array}$ & 0 & 96 & Prof. & 20 \\
\hline
\end{tabular}




\begin{tabular}{|c|c|c|c|c|c|c|}
\hline \multirow{2}{*}{ Nombre } & \multirow{2}{*}{ Autor/es } & \multirow{2}{*}{ Descripción: } & \multicolumn{2}{|c|}{ Edad } & \multirow{2}{*}{ 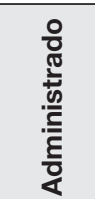 } & \multirow{2}{*}{$\frac{\circ}{\varrho_{0}^{\circ}}$} \\
\hline & & & 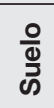 & 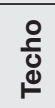 & & \\
\hline $\begin{array}{l}\text { 3.- Bayley III } \\
\text { Screening Test, } \\
\text { (BINS). }\end{array}$ & (Bayley, 2006). & $\begin{array}{l}\text { Herramienta de administración directa. } \\
\text { Compuesta de series de } 6 \text { ítems que evalúan } \\
\text { funciones neurológicas básicas, funciones } \\
\text { receptivas (input visual, táctil y auditivo), } \\
\text { funciones expresivas (habilidades orales, motoras } \\
\text { finas y gruesas) y procesos cognitivos. Los } \\
\text { resultados se expresan en riesgo bajo, moderado } \\
\text { o alto. }\end{array}$ & 1 & 42 & Mixta & $15-25$ \\
\hline $\begin{array}{l}\text { 4.- Brigance } \\
\text { Early Childhood } \\
\text { Screens. }\end{array}$ & $\begin{array}{l}\text { (Brigance y } \\
\text { Glascoe, 2002). }\end{array}$ & $\begin{array}{l}\text { Es una reconfiguración de los otros dos } \\
\text { instrumentos anteriores (earlier Infant and } \\
\text { Toddler Screen-II y Early Preschool Screen-II). } \\
\text { Herramientas de administración directa que } \\
\text { evalúa conocimiento general y comprensión, } \\
\text { lenguaje expresivo, habilidades motoras } \\
\text { gruesas y finas, conocimientos pre-académicos, } \\
\text { habilidades socio-emocionales y autoayuda. }\end{array}$ & 0 & 35 & Prof. & 15 \\
\hline $\begin{array}{l}\text { 5.- Child } \\
\text { Development } \\
\text { Inventory. }\end{array}$ & $\begin{array}{l}\text { (Ireton,1992; } \\
\text { Ireton y } \\
\text { Glascoe, 1995). }\end{array}$ & $\begin{array}{l}\text { Cuestionario para detectar problemas en el } \\
\text { desarrollo social, motor, lenguaje, auto-ayuda, } \\
\text { inicio de la lecto-escritura y numeración y en } \\
\text { general síntomas de problemas conductuales. } \\
\text { Incluye una escala de desarrollo general que } \\
\text { contestan los/as padres/madres. }\end{array}$ & 12 & 72 & Mixta & $30-50$ \\
\hline $\begin{array}{l}\text { 6.- Child } \\
\text { Development } \\
\text { Review. }\end{array}$ & (Ireton, 2004). & $\begin{array}{l}\text { Está dividido en dos secciones "Parent } \\
\text { Questionaire (CDR-PQ) y el "Child Development } \\
\text { Chart". El primero lo contestan los/as padres/ } \\
\text { madres y el segundo los/las profesionales. Evalúa } \\
\text { habilidades de auto-ayuda, motoras, sociales y } \\
\text { de lenguaje. }\end{array}$ & 18 & 60 & Mixta & $10-20$ \\
\hline $\begin{array}{l}\text { 7.- Cognitive } \\
\text { Abilities Scale } \\
\text { 2nd ed. } \\
\text { (CAS-2). }\end{array}$ & $\begin{array}{l}\text { (Johnson } \\
\text { y Bradley- } \\
\text { Johnson,2002; } \\
\text { Bradley- } \\
\text { Johnson y } \\
\text { Johnson, 2001). }\end{array}$ & $\begin{array}{l}\text { Herramienta de evaluación diseñada para la } \\
\text { identificación temprana de niños/as con retrasos } \\
\text { cognitivos. Hay dos formas, infantil (79ítems) y } \\
\text { Preescolar ( } 88 \text { ítems). La forma infantil se divide } \\
\text { en tres secciones: la exploración de los objetos, } \\
\text { de comunicación, de iniciación y de imitación } \\
\text { de actividades. El formulario de pre-escolar } \\
\text { se divide en cinco secciones: el lenguaje oral, } \\
\text { la lectura, matemáticas, escritura y facilitar } \\
\text { comportamientos. }\end{array}$ & 3 & 47 & Prof. & $20-30$ \\
\hline $\begin{array}{l}\text { 8.- } \\
\text { Developmental } \\
\text { Activities } \\
\text { Screening } \\
\text { Inventory-II } \\
\text { (DASI-II). }\end{array}$ & $\begin{array}{l}\text { (Fewell y } \\
\text { Langley, 1984). }\end{array}$ & $\begin{array}{l}\text { Diseñado para la detección temprana de retraso } \\
\text { en el desarrollo, con especial énfasis en los/as } \\
\text { niños/as pequeños con trastorno del lenguaje } \\
\text { cuyas habilidades cognitivas no pueden ser } \\
\text { examinadas con precisión con una herramienta } \\
\text { que requiere que el niño/a siga las instrucciones } \\
\text { habladas. Instrucciones DASI-II puede ser verbal } \\
\text { o visual. Hay } 67 \text { elementos que se pueden } \\
\text { administrar en una o dos configuraciones. } \\
\text { Las tareas se organizan en } 15 \text { categorías de } \\
\text { habilidades que incluyen insensibilidad sensorial, } \\
\text { relaciones de causa-efecto, la causalidad, la } \\
\text { memoria y el razonamiento. }\end{array}$ & 1 & 66 & Prof. & $25-30$ \\
\hline
\end{tabular}




\begin{tabular}{|c|c|c|c|c|c|c|}
\hline \multirow{2}{*}{ Nombre } & \multirow{2}{*}{ Autor/es } & \multirow{2}{*}{ Descripción: } & \multicolumn{2}{|c|}{ Edad } & \multirow{2}{*}{ 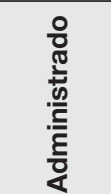 } & \multirow{2}{*}{ 을 } \\
\hline & & & $\frac{\circ}{\stackrel{0}{J}}$ & 응 & & \\
\hline $\begin{array}{l}\text { 9.- } \\
\text { Developmental } \\
\text { Assessment of } \\
\text { Young Children, } \\
\text { 2nd ed. (DAYC- } \\
\text { 2). }\end{array}$ & $\begin{array}{l}\text { (Voress y } \\
\text { Maddox, 1998). }\end{array}$ & $\begin{array}{l}\text { Administrada individualmente en la primera } \\
\text { infancia .Tiene como objetivos principales ayudar } \\
\text { a identificar a los/as niños/as en el área cognitiva, } \\
\text { comunicativa o social-emocional, físico, } \\
\text { habilidades de conducta adaptativa. También } \\
\text { puede utilizarse como seguimiento de programas } \\
\text { de intervención. }\end{array}$ & 0 & 71 & Prof. & $10-20$ \\
\hline $\begin{array}{l}\text { 10- Denver } \\
\text { Developmental } \\
\text { Screening Test II } \\
\text { (DDST-II). }\end{array}$ & $\begin{array}{l}\text { (Frankenburg } \\
\text { et al.1971; } \\
\text { Frankenburg y } \\
\text { Dodds,1967). }\end{array}$ & $\begin{array}{l}\text { Esta herramienta consta de } 125 \text { ítems que } \\
\text { evalúa } 4 \text { áreas del funcionamiento del niño/a: } \\
\text { motora, adaptativa, personal-social y habilidades } \\
\text { comunicativas. }\end{array}$ & 0 & 72 & Prof. & $20-30$ \\
\hline $\begin{array}{l}\text { 11.- } \\
\text { Developmental } \\
\text { Observation } \\
\text { Checklist } \\
\text { System (DOCS). }\end{array}$ & $\begin{array}{l}\text { (Hresko et al. } \\
\text { 1994). }\end{array}$ & $\begin{array}{l}\text { El instrumento es completado principalmente por } \\
\text { el profesional aunque existe una parte que es } \\
\text { completada por la familia. Consta de } 540 \text { ítems } \\
\text { en total pero dependiendo de la edad del niño/a } \\
\text { se completa una cantidad específica. Evalúa } \\
\text { desarrollo general (lenguaje, motor, social y } \\
\text { desarrollo cognitivo), comportamiento del niño/a } \\
\text { y apoyo y estrés de los/as padres/madres. }\end{array}$ & 0 & 72 & Mixta & 30 \\
\hline $\begin{array}{l}\text { 12.- } \\
\text { Developmental } \\
\text { Profile } 3 \text { (DP-3). }\end{array}$ & (Alpern, 2007). & $\begin{array}{l}\text { Permite evaluar de forma rápida las cinco } \\
\text { principales áreas del desarrollo infantil } \\
\text { (Cognición, Motricidad, Socioemocional, } \\
\text { Comunicación y Conducta adaptativa) y ofrece } \\
\text { un índice global de desarrollo del niño/a. Dispone } \\
\text { de dos formas de aplicación alternativas: } \\
\text { mediante entrevista a los/as padres/madres o } \\
\text { mediante un cuestionario que responden estos } \\
\text { de forma independiente. }\end{array}$ & 0 & 155 & Padres & $20-40$ \\
\hline $\begin{array}{l}\text { 13.- Early } \\
\text { Learning } \\
\text { Accomplishment } \\
\text { Profile (E-LAP). }\end{array}$ & $\begin{array}{l}\text { (Hardin y } \\
\text { Peisner, 2001). }\end{array}$ & $\begin{array}{l}\text { El E-LAP es una herramienta de evaluación } \\
\text { referida a criterios. Se puede utilizar para } \\
\text { identificar a los/as niños/as pequeños que } \\
\text { necesitan una remisión para una evaluación del } \\
\text { desarrollo. Los ítems provienen de una amplia } \\
\text { gama de instrumentos de detección y evaluación } \\
\text { estandarizados. El sistema LAP se centra en } \\
\text { las cinco áreas del desarrollo principales: motor } \\
\text { (grueso-fino), auto-ayuda (adaptación), lenguaje } \\
\text { (comunicación), cognitivos y de funcionamiento } \\
\text { socio-emocional. }\end{array}$ & 0 & 36 & Prof. & $12-15$ \\
\hline $\begin{array}{l}\text { 14.- First Step: } \\
\text { Screening Test } \\
\text { for Evaluating } \\
\text { Preschoolers. }\end{array}$ & $\begin{array}{l}\text { (Roxswell,1993; } \\
\text { Miler, 1993). }\end{array}$ & $\begin{array}{l}\text { Instrumento que evalúa directamente las áreas: } \\
\text { cognitivo, comunicación y motor. Se compone } \\
\text { también de dos "checklists", una de ítems socio- } \\
\text { emocionales basadas en las observaciones del } \\
\text { examinador y otra, que completan los padres } \\
\text { sobre el comportamiento adaptativo de su hijo/a. }\end{array}$ & 33 & 74 & Mixto & $15-20$ \\
\hline $\begin{array}{l}\text { 15.- Infant } \\
\text { Development } \\
\text { Inventory (IDI). }\end{array}$ & $\begin{array}{l}\text { (Creighton y } \\
\text { Sauve, 1988). }\end{array}$ & $\begin{array}{l}\text { Cuestionario que completan los/as padres/ } \\
\text { madres o cuidadores que evalúa el desarrollo a } \\
\text { través de las áreas: social, autoayuda, motora, y } \\
\text { habilidades de la comunicación y el lenguaje. }\end{array}$ & 0 & 18 & Padres & $5-10$ \\
\hline
\end{tabular}




\begin{tabular}{|c|c|c|c|c|c|c|}
\hline \multirow[b]{2}{*}{ Nombre } & \multirow[b]{2}{*}{ Autor/es } & \multirow[b]{2}{*}{ Descripción: } & \multicolumn{2}{|c|}{ Edad } & \multirow{2}{*}{ 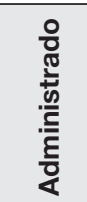 } & \multirow{2}{*}{$\frac{\stackrel{\circ}{\varepsilon}}{\stackrel{\varrho}{\varrho}}$} \\
\hline & & & $\frac{\circ}{\frac{0}{\varrho}}$ & 응 & & \\
\hline $\begin{array}{l}\text { 16.- Individual } \\
\text { Growth and } \\
\text { Development } \\
\text { Indicators } \\
\text { (GDIs). }\end{array}$ & $\begin{array}{l}\text { (Greenwood et } \\
\text { al. 2005). }\end{array}$ & $\begin{array}{l}\text { Conjunto de medidas diseñadas y validadas } \\
\text { para su uso por profesionales. Evalúa las áreas: } \\
\text { comunicación, movimiento, resolución de } \\
\text { problemas, la interacción social. A diferencia de } \\
\text { las pruebas estandarizadas que se administran } \\
\text { con poca frecuencia, IGDI de están diseñados } \\
\text { para ser utilizado en varias ocasiones por los } \\
\text { médicos para estimar la "tasa de crecimiento" de } \\
\text { cada niño/a a través del tiempo. }\end{array}$ & 0 & 36 & Prof. & 40 \\
\hline $\begin{array}{l}\text { 17.-Infant- } \\
\text { Toddler } \\
\text { Developmental } \\
\text { Assessment } \\
\text { (IDA). }\end{array}$ & (Erikson, 1996). & $\begin{array}{l}\text { Este instrumento realiza un cribado del } \\
\text { funcionamiento del desarrollo del niño/a en } \\
\text { varias áreas: motricidad fina y gruesa, relación } \\
\text { con los objetos inanimados (cognitiva), lenguaje/ } \\
\text { comunicación, autoayuda, relaciones con las } \\
\text { personas, emociones, sentimientos e imitación. }\end{array}$ & 0 & 42 & Prof. & - \\
\hline $\begin{array}{l}\text { 18.- Infant- } \\
\text { Toddler } \\
\text { and Family } \\
\text { Instrument (ITFI). }\end{array}$ & $\begin{array}{l}\text { (Provence y } \\
\text { Apfel, 2001). }\end{array}$ & $\begin{array}{l}\text { ITFI permite a los proveedores de servicios } \\
\text { familiares reunir información e impresiones } \\
\text { acerca de un niño/a y la familia y su entorno } \\
\text { familiar, ayudando a los proveedores a decidir } \\
\text { si se necesitan más referencias y servicios. } \\
\text { Evalúa las siguientes áreas: motricidad gruesa } \\
\text { y fina, social y emocional, del lenguaje, de } \\
\text { afrontamiento y auto-ayuda. }\end{array}$ & 6 & 36 & Prof. & - \\
\hline $\begin{array}{l}\text { 19.- Kent } \\
\text { Inventory of } \\
\text { Developmental } \\
\text { Skills-3rd } \\
\text { ed.(KIDS). }\end{array}$ & $\begin{array}{l}\text { (Reuter et al. } \\
\text { 1996; Reuter y } \\
\text { Gruber, 2000). }\end{array}$ & $\begin{array}{l}\text { Cuestionario que se completa por la familia o el } \\
\text { cuidador principal, basado en las observaciones } \\
\text { de comportamiento en diferente rango de } \\
\text { condiciones. Consta de } 252 \text { ítems, dirigidos a } \\
\text { evaluar las áreas: motoras, autoayuda, cognitivo, } \\
\text { comunicación y habilidades sociales. }\end{array}$ & 0 & 15 & Prof. & 45 \\
\hline $\begin{array}{l}\text { 20.-Minnesota } \\
\text { Child } \\
\text { Development } \\
\text { Inventory } \\
\text { (MCDI). }\end{array}$ & $\begin{array}{l}\text { (Ireton et al. } \\
\text { 1977). }\end{array}$ & $\begin{array}{l}\text { MCDI: Esta escala es un cuestionario de padres/ } \\
\text { madres, está compuesta por } 320 \text { ítems. Evalúa } \\
\text { las siguientes áreas: desarrollo general, la } \\
\text { motricidad gruesa, motricidad fina, lenguaje } \\
\text { expresivo, comprensión conceptual, situación de } \\
\text { comprensión, de autoayuda, y personal-social. } \\
\text { Esta prueba está diseñada para complementar } \\
\text { una entrevista con los padres cuando se han } \\
\text { planteado cuestiones de retraso en el desarrollo. }\end{array}$ & 12 & 72 & Prof. & $30-50$ \\
\hline $\begin{array}{l}\text { 21.-Prueba } \\
\text { Nacional de } \\
\text { Pesquisa } \\
\text { (PRUNAPE). }\end{array}$ & $\begin{array}{l}\text { (Leiarraga et al., } \\
\text { 2005). }\end{array}$ & $\begin{array}{l}\text { Es una prueba desarrollada en Argentina. La } \\
\text { prueba consta de setenta y nueve pautas } \\
\text { madurativas pertenecientes a las siguientes } \\
\text { áreas: motricidad fina y gruesa, lenguaje y } \\
\text { personal-social. La prueba consiste en solicitar } \\
\text { al niño/a que realice una serie de ejercicios con } \\
\text { papel y lápiz, cubos, tarjetas dibujadas, que } \\
\text { varían según la edad. }\end{array}$ & 0 & 72 & Prof. & 15 \\
\hline
\end{tabular}




\begin{tabular}{|c|c|c|c|c|c|c|}
\hline \multirow{2}{*}{ Nombre } & \multirow{2}{*}{ Autor/es } & \multirow{2}{*}{ Descripción: } & \multicolumn{2}{|c|}{ Edad } & \multirow{2}{*}{ 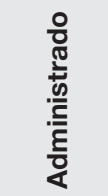 } & \multirow{2}{*}{ 을 } \\
\hline & & & $\begin{array}{l}\frac{0}{0} \\
\stackrel{\varpi}{\partial}\end{array}$ & 옹 & & \\
\hline $\begin{array}{l}\text { 22.- Parents' } \\
\text { Evaluation of } \\
\text { Developmental } \\
\text { Status (PEDS). }\end{array}$ & (Glascoe, 1998). & $\begin{array}{l}\text { Cuestionarios para padres/madres que a través } \\
\text { de } 10 \text { ítems pregunta sobre las preocupaciones } \\
\text { de los padres y evalúa el área cognitiva, lenguaje } \\
\text { expresivo y receptivo, habilidades motoras fina } \\
\text { y gruesa, comportamiento y habilidades socio- } \\
\text { emocionales. }\end{array}$ & 0 & 96 & Padres & 5 \\
\hline $\begin{array}{l}\text { 23.- Parents' } \\
\text { Evaluation of } \\
\text { Developmental } \\
\text { Status } \\
\text { Developmental } \\
\text { Milestones } \\
\text { (PEDS-DM). }\end{array}$ & $\begin{array}{l}\text { (Brothers et al. } \\
\text { 2008). }\end{array}$ & $\begin{array}{l}\text { Herramienta para detectar el retraso en el } \\
\text { desarrollo. Sustituye la lista de chequeo } \\
\text { convencional. Intenta monitorizar el progreso } \\
\text { del niño/a a lo largo de la infancia. El PEDS-DM } \\
\text { contempla un total de } 20 \text { grupos de edad para } \\
\text { cada uno dispone de un cuestionario diferente. }\end{array}$ & 0 & 96 & Padres & $15-20$ \\
\hline
\end{tabular}

Fuente: Elaboración propia.

\section{Evaluación de la calidad de las herramientas}

Entre las herramientas y pruebas detectadas se encontraron gran diversidad de tipos y formas de administración. Desde cuestionarios de investigación hasta herramientas diagnósticas aplicadas con fines de detección. En este sentido, es de destacar que se está generalizando la práctica de los autoinformes sobre el desarrollo de los/as niños/as ofrecido por los/as padres/ madres. Esta práctica es más general, cuanto más bajo es el suelo de aplicación y cuanto menos observable es la conducta sobre la que se hace la evaluación, demostrando su eficacia como "screening” sobre todo en niños/ as pequeños y sobre el desarrollo inmediato (Glascoe, I997).

El criterio de calidad para estos test o cuestionarios se basa en la fiabilidad, validez, sensibilidad y especificidad y en medidas derivadas de estas (Camp, 2006; Johnson y Marlow, 2006). No obstante, existen otros datos que aunque menos objetivos pueden hacer que un sistema sea mejor valorado que otro. Así, la simplicidad y brevedad de administración, el bajo coste de la misma, la interoperabilidad de los resultados, la accesibilidad a los sistemas, la adaptación a otras lenguas y culturas, son otros datos que deben tenerse en cuenta a la hora de seleccionar un instrumento u otro.

Con la finalidad de evaluar los instrumentos analizados se construyó una tabla con la valoración de los diferentes criterios de calidad, teniendo en cuenta tanto las características de las normas de administración como de los resultados psicométricos recogidos en diferentes publicaciones, siguiendo en este sentido las recomendaciones del SAC ${ }^{2}$ y otros (Terwee et al. 2007).

La evaluación de calidad se realizó aplicando los criterios descritos en la Tabla 2. Se sumaron todas las puntuaciones obteniendo una puntuación total.

2. SAC: Scientific Advisory Committee of the Medical Outcomes Trust (Aaronson et al., 2002) 


\begin{tabular}{|c|c|c|}
\hline INDICADOR & DEFINICIÓN & CRITERIO DE CALIDAD \\
\hline \multicolumn{3}{|l|}{ 1. Suelo y Techo } \\
\hline Suelo & Edad mínima de aplicación & $\begin{array}{l}\text { 3: Aplicación por debajo de los tres primeros meses de vida. } \\
\text { 2: Aplicación desde los seis meses de vida. } \\
\text { 1: Aplicación desde el año de vida. } \\
\text { 0: Aplicación después del primer año de vida. }\end{array}$ \\
\hline Techo & Edad máxima de aplicación & $\begin{array}{l}\text { No se valora ningún techo específico. Aunque por la naturaleza } \\
\text { de la información no debería ser superior a los } 36-48 \text { meses. }\end{array}$ \\
\hline \multicolumn{3}{|l|}{ 2. Fiabilidad } \\
\hline Consistencia Interna & $\begin{array}{l}\text { Grado en que los diferentes ítems } \\
\text { o sub-pruebas miden el mismo } \\
\text { constructo. Generalmente se } \\
\text { evalúa mediante el coeficiente } \\
\text { Alpha de Cronbach (correlación } \\
\text { pares-impares) }\end{array}$ & $\begin{array}{l}\text { 3: Alfa entre } 0,70 \text { y } 0,95 \text {. } \\
\text { 2: Alfa menor a } 0,70 \text { a pesar de diseño y método adecuado. } \\
\text { 1: Diseño dudoso sin resultados significativos. } \\
\text { 0: Información no encontrada. }\end{array}$ \\
\hline $\begin{array}{l}\text { Estabilidad de la } \\
\text { medida (Test-Retest) }\end{array}$ & $\begin{array}{l}\text { Grado en que las puntuaciones } \\
\text { de medidas repetidas son } \\
\text { semejantes o consistentes. Se } \\
\text { evalúa mediante Correlación de } \\
\text { Pearson. }\end{array}$ & $\begin{array}{l}\text { 3: Coeficiente entre } 0,70 \text { y } 0,95 \text {. } \\
\text { 2: Coeficiente menor a } 0,70 \text { a pesar de diseño y método } \\
\text { adecuado. } \\
\text { 1: Diseño dudoso sin resultados significativos. } \\
\text { 0: Información no encontrada. }\end{array}$ \\
\hline \multicolumn{3}{|l|}{ 3. Validez } \\
\hline $\begin{array}{l}\text { Validez de Criterio o } \\
\text { Concurrente }\end{array}$ & $\begin{array}{l}\text { Grado de ajuste entre la } \\
\text { puntuación del cuestionario y un } \\
\text { Gold Standard. }\end{array}$ & $\begin{array}{l}\text { 3: Correlación con la Gold Standard superiores a } 0,70 . \\
\text { 2: Correlación con Gold Standard menor a } 0,70 \text { a pesar de } \\
\text { diseño y método adecuado. } \\
\text { 1: No hay argumentos convincentes sobre la adecuación de la } \\
\text { Gold Standard o el diseño o el método es dudoso. } \\
\text { 0: Información no encontrada. }\end{array}$ \\
\hline \multicolumn{3}{|c|}{ 4. Capacidad diagnóstica } \\
\hline Sensibilidad & $\begin{array}{l}\text { Proporción de sujetos evaluados } \\
\text { mediante la herramienta de } \\
\text { cribado con resultado positivo } \\
\text { que finalmente se confirma su } \\
\text { diagnóstico. }\end{array}$ & $\begin{array}{l}\text { 3: Entre } 0,70 \text { y } 0,95 . \\
\text { 2: Menor a } 0,70 \text { a pesar de diseño y método adecuado. } \\
\text { 1: Diseño dudoso sin datos explícitos. } \\
0: \text { Información no encontrada. }\end{array}$ \\
\hline Especificidad & $\begin{array}{l}\text { Proporción de sujetos evaluados } \\
\text { mediante la herramienta de } \\
\text { cribado con resultado negativo } \\
\text { que finalmente se descarta el } \\
\text { diagnóstico. }\end{array}$ & $\begin{array}{l}\text { 3: Entre } 0,70 \text { y } 0,95 . \\
\text { 2: Menor a } 0,70 \text { a pesar de diseño y método adecuado. } \\
\text { 1: Diseño dudoso sin datos explícitos. } \\
\text { 0: Información no encontrada. }\end{array}$ \\
\hline \multicolumn{3}{|l|}{ 5.- Otros } \\
\hline Otros & $\begin{array}{l}\text { Facilidad de uso, interpretabilidad } \\
\text { de los resultados, accesibilidad } \\
\text { de la herramienta, existe versión } \\
\text { en castellano. }\end{array}$ & $\begin{array}{l}\text { 3: Existe versión en castellano y resulta fácil de administrar, } \\
\text { interpretable y accesible. } \\
\text { 2: Cumple solo parcialmente alguno de los diferentes criterios. } \\
\text { 1: Cumple de forma dudosa alguno de los criterios. } \\
\text { 0: No cumple ningún criterio. }\end{array}$ \\
\hline
\end{tabular}

Fuente: Elaboración propia. 


\section{Resultados}

En la Tabla 3 se presentan los resultados de aplicar los criterios de calidad a los 23 instrumentos de cribado detectados en el proceso de revisión. Es de destacar la falta de información psicométrica en general. En particular, la referente a la validez y al valor diagnóstico (sensibilidad, especificidad y valores predictivos positivos y negativos), cuando no un excesivo número de estudios con valores diferentes en función del criterio diagnóstico, tamaño de la muestra, tiempo de la aplicación, etc.

Con respecto al primer criterio (suelo de la prueba) como se trata de uno de los criterios de inclusión en la búsqueda sistemática, se observa que todas las herramientas tienen una puntuación alta. Respecto a la fiabilidad como consistencia interna es el dato psicométrico más frecuente, la valoración obtenida cuando se aporta este dato suele ser alta. De la misma forma ocurre con la fiabilidad como estabilidad de la medida (test-retest), que aunque son muy pocas las pruebas en las que se han encontrado resultados, cuando se han localizado suelen ser medio o medio-alto. No se ha hallado información sobre la fiabilidad inter-jueces de los instrumentos.

El punto más débil en las herramientas analizadas, es la poca o ninguna información que se aporta sobre su validez. Son estudios a posteriori comparando resultados de diferentes instrumentos los que aportan, en todo caso, la información sobre la validez. En este sentido, los datos hacen referencia exclusivamente a la validez de criterio o validez concurrente. Se entiende que, para determinar la validez de constructo o valor predictivo de los ítems en la detección de trastornos concretos, se hacen necesarios estudios longitudinales con muestras muy elevadas y consecuentemente con un alto coste económico.

Al ordenar esta puntuación total se observa que existen tres pruebas que se encuentran dentro del primer cuartil y ocho más en el segundo cuartil (ver Tabla 3). Se trata de las formas cortas o screening de las escalas Bayley (Bayley III Screening Test) y Battelle (Battelle Developmental Inventory Screening Test) y del sistema de detección Ages and Stages Questionnaires (ASQ-3).

La primera de ellas, Bayley III Screening Test (Bayley, 2006) utiliza una forma de administración mixta, parte de los ítems se observan directamente sobre el niño/a y parte, se recoge la información mediante autoinforme de los/as padres/madres. Los resultados permiten clasificar a los/as niños/as como "competente", "emergente" o "de riesgo". Estas categorías se determinaron en base a la puntuación alcanzada por los/as niños/as en la aplicación de la BSID$\mathrm{III}^{3}$. Aunque existen versiones en castellano, la traducción se ha realizado para población hispana norteamericana y se hace necesaria una adaptación al castellano de España. Por otra parte, no existe estudio de tipificación de la BSTIII ${ }^{4}$ en población española.

La segunda prueba, Ages and Stages Questionnaires (ASQ-3), es un sistema de detección compuesto por 2 I cuestionarios, uno específico para cada corte de edad desde los 2 meses hasta los 60 meses. Los cuestionarios pueden ser cumplimentados directamente por los/as padres/madres durante la revisión pediátrica, enviados por correo u "online". Cada cuestionario cuenta con unos pequeños datos demográficos y treinta preguntas sobre el desarrollo del niño/a dividido en cinco dominios. Si la puntuación obtenida es inferior a un punto de corte significa que es necesaria una evaluación más especializada. La validación de este sistema de detección se realizó con las escalas Battelle Developmental Inventory II y Bayley BSID-II obteniendo muy buenos resultados (Gollenberg et al. 2010). Este sistema ha sido adaptado a numerosos países aunque en muy pocos estudios de adaptación se han re-examinado sus propiedades psicométricas (Kerstjems et al. 2009). La versión española ha

\footnotetext{
3. Bayley Scales of Infant and Toddler Development. Edition III.

4. Bayley Screening Test III.
} 


\begin{tabular}{|c|c|c|c|c|c|c|c|c|c|}
\hline \multirow{2}{*}{\multicolumn{2}{|c|}{ Instrumentos }} & \multirow{3}{*}{$\begin{array}{l}\text { Edad } \\
\text { Suelo } \\
3,00 \\
\end{array}$} & \multicolumn{2}{|c|}{ Fiabilidad } & \multirow{3}{*}{\begin{tabular}{c|} 
Validez \\
2,00 \\
\end{tabular}} & \multicolumn{2}{|c|}{ Diagnóstico } & \multirow{3}{*}{$\begin{array}{l}\text { Otros } \\
3,00 \\
\end{array}$} & \multirow{3}{*}{$\begin{array}{l}\text { Total } \\
20,00\end{array}$} \\
\hline & & & \multirow{2}{*}{$\begin{array}{c}\text { Consistencia } \\
3,00 \\
\end{array}$} & \multirow{2}{*}{$\begin{array}{l}\text { Re- } \\
\text { Test } \\
3,00\end{array}$} & & \multirow{2}{*}{$\begin{array}{c}\text { Sensibilidad } \\
3,00\end{array}$} & \multirow{2}{*}{$\begin{array}{c}\text { Especificidad } \\
3,00 \\
\end{array}$} & & \\
\hline & 3.- Bayley III Screening Test. & & & & & & & & \\
\hline 孛 & 1.- Ages and Stages Questionnaires. & 3,00 & 3,00 & 3,00 & 3,00 & 2,00 & 2,00 & 3,00 & 19,00 \\
\hline$\stackrel{\circ}{\circ}$ & $\begin{array}{l}\text { 2.- Battelle Developmental Inventory } \\
\text { Screening Test. }\end{array}$ & 3,00 & 3,00 & & 3,00 & 3,00 & 3,00 & 3,00 & 18,00 \\
\hline \multirow{8}{*}{ 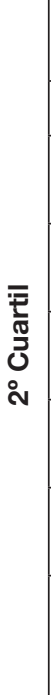 } & $\begin{array}{l}\text { 14.- First STEp: Screening Test for } \\
\text { Evaluating Preschoolers. }\end{array}$ & 3,00 & 3,00 & & 3,00 & 2,00 & 2,00 & 2,00 & 15,00 \\
\hline & 5.- Child Development Inventory. & 2,00 & 3,00 & & 2,00 & 2,00 & 3,00 & 2,00 & 14,00 \\
\hline & $\begin{array}{l}\text { 13.- Early Learning Accomplishment } \\
\text { Profile. }\end{array}$ & 3,00 & 3,00 & & 2,00 & 3,00 & 2,00 & 1,00 & 14,00 \\
\hline & $\begin{array}{l}\text { 19.- Kent Inventory of Developmental } \\
\text { Skills. }\end{array}$ & 3,00 & 3,00 & 3,00 & 3,00 & & & 2,00 & 14,00 \\
\hline & $\begin{array}{l}\text { 22.- Parents' Evaluation of } \\
\text { Developmental Status. } \\
\end{array}$ & 3,00 & 3,00 & & 1,00 & 2,00 & 2,00 & 3,00 & 14,00 \\
\hline & $\begin{array}{l}\text { 4.- Brigance Early Childhood } \\
\text { Screens. }\end{array}$ & 3,00 & 2,00 & & 2,00 & 2,00 & 2,00 & 2,00 & 13,00 \\
\hline & $\begin{array}{l}\text { 8.- Developmental Activities } \\
\text { Screening Inventory-II. }\end{array}$ & 3,00 & 3,00 & 3,00 & 2,00 & & & 1,00 & 12,00 \\
\hline & $\begin{array}{l}\text { 23.- Parents' Evaluation } \\
\text { of Developmental Status } \\
\text { Developmental Milestones. }\end{array}$ & 3,00 & & & 1,00 & 2,00 & 3,00 & 2,00 & 11,00 \\
\hline \multirow{7}{*}{ 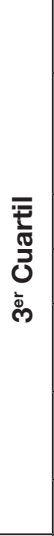 } & $\begin{array}{l}\text { 11.- Developmental Observation } \\
\text { Checklist System. }\end{array}$ & 3,00 & 3,00 & & 2,00 & & & 2,00 & 10,00 \\
\hline & $\begin{array}{l}\text { 16.- Individual Growth and } \\
\text { Development Indicators (IGDIs) for } \\
\text { Infants and Toddlers. }\end{array}$ & 3,00 & 3,00 & & 2,00 & & & 2,00 & 10,00 \\
\hline & 21.- Prueba Nacional de Pesquisa. & 3,00 & & & & 2,00 & 3,00 & 2,00 & 10,00 \\
\hline & 15.- Infant Development Inventory. & 3,00 & & & & 2,00 & 2,00 & 2,00 & 9,00 \\
\hline & $\begin{array}{l}\text { 20.- Minnesota Child Development } \\
\text { Inventory. }\end{array}$ & 1,00 & & & & 3,00 & 3,00 & 2,00 & 9,00 \\
\hline & $\begin{array}{l}\text { 10.- Denver Developmental } \\
\text { Screening Test. }\end{array}$ & 3,00 & & & & 2,00 & 1,00 & 2,00 & 8,00 \\
\hline & 6.- Child Development Review. & 2,00 & & & & 1,00 & 2,00 & 2,00 & 7,00 \\
\hline \multirow{5}{*}{ 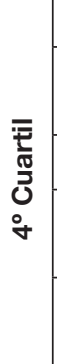 } & 7.- Cognitive Abilities Scale. & 2,00 & & & 2,00 & & & 1,00 & 5,00 \\
\hline & $\begin{array}{l}\text { 9.- Developmental Assessment of } \\
\text { Young Children. }\end{array}$ & 3,00 & & & & & & 1,00 & 4,00 \\
\hline & 12.- Developmental Profile 3. & 3,00 & & & & & & 1,00 & 4,00 \\
\hline & $\begin{array}{l}\text { 17.- Infant-Toddler Developmental } \\
\text { Assessment. }\end{array}$ & 3,00 & & & & & & 1,00 & 4,00 \\
\hline & $\begin{array}{l}\text { 18.- Infant-Toddler and Family } \\
\text { Instrument. }\end{array}$ & 2,00 & & & & & & 1,00 & 3,00 \\
\hline
\end{tabular}


sido traducida para la población hispana de USA y solo disponemos de datos psicométricos de la adaptación de los cuestionarios de 9, I 8 y 30 meses (Pomés, 20I2).

La tercera de ellas, Battelle Developmental Inventory Screening Test (BDIST), (Newborg, 2005), consiste en 96 ítems extraídos de la BDI-II. Evalúa las áreas de desarrollo motor fino y grueso, conducta adaptativa, socioemocional, lenguaje comprensivo y expresivo y estilos cognitivos. El tiempo de administración oscila entre los Io minutos en los/as niños/ as más pequeños y los 30 en los/as niños/as mayores. Existe cierta preocupación sobre las propiedades psicométricas dado que en origen no formaba parte del estudio de normalización de la prueba completa (Bliss y Newborg, 2007). Las normas de aplicación son las mismas que en la escala general por lo que se puede considerar como una forma breve de la BDI-II diseñada exclusivamente para detectar a los/as niños/as que precisan una evaluación de mayor amplitud y profundidad.

Con respecto a las ocho pruebas que quedan ordenadas en el segundo cuartil destacamos, Parents' Evaluation of Developmental Status (PEDS), (Glascoe, I998) y el Parents' Evaluation of Developmental Status Developmental Milestones (PEDS-DM), (Easterbrooks y Goldberg, I984; Brothers et al., 2008) por la originalidad de la idea de detectar el trastorno por medio de la preocupación de los propios padres (PEDS) o por el conocimiento de los hitos evolutivos (PEDS-DM). El primero ha sido comparado con numerosos sistemas de detección (CDI, Brigance Screens, BDIST, etc) con buenos resultados (Halle et al. 20II). En la actualidad ya existe versión electrónica que clasifica a los/as niños/as como de alto, moderado o bajo riesgo. El PEDS-DM puede utilizarse, de forma independiente o complementaria, para realizar un seguimiento longitudinal a lo largo del desarrollo del niño/a. Consiste en 20 cuestionarios distribuidos por edad desde el nacimiento hasta los 8 años aproximadamente.

El Child Development Inventory (CDI) es un cuestionario para la detección y evaluación de niños/as en los que existe preocupación por su desarrollo. Consiste en 300 ítems que los padres/ madres pueden observar en sus hijos/as en situaciones cotidianas. La puntuación obtenida se traslada a un perfil donde se contrasta con la puntuación promedio de su edad. Se considera problemática una puntuación por debajo de 2 desviaciones de la media de su edad. El CDI es el resultado de 30 años de investigación y experiencia clínica con el Inventario Minnesota Desarrollo Infantil (MCDI), (Ireton y Glascoe, I995).

Por último, destacar también que el test de Denver, prueba clásica, obtiene una puntuación baja quedando en el tercer cuartil. Su puntuación se justifica por la falta de estudios de validez, tanto de constructo como diagnóstico, desconociendo datos de su sensibilidad y especificidad (Frankenburg et al. I992). Otros estudios han demostrado que la escala tiene una buena sensibilidad pero baja especificidad (Glascoe et al. I992; Hamilton, 2006).

\section{Discusión y Conclusión}

El cribado del desarrollo es un procedimiento de evaluación breve diseñado para identificar a niños que deberían recibir una evaluación más exhaustiva (Meisels y Provence, I989). Para poder obtener una aplicación universal se debería conseguir un instrumento que suponga bajo coste en su aplicación. Para ello, se ha demostrado que los cuestionarios de autoinformes de los/as padres/madres tienen suficiente validez y fiabilidad como para ser considerados como sistemas de nivel I. El uso de herramientas de cribado por los padres, aunque claramente reduce coste, genera una preocupación general sobre la fiabilidad de las respuestas de los padres respecto al desarrollo de sus hijos (Sheehan, I988). Esta preocupación dio pie a muchas investigaciones alrededor de su validez. En la mayoría de las investigaciones se informa de un acuerdo consistente entre las evaluaciones de profesionales y padres, en 
particular cuando estos últimos respondían a cuestiones sobre el desarrollo actual (Squires et al. I998). Otra ventaja práctica que se les atribuye a este tipo de cuestionarios es que las madres están más preocupadas por el comportamiento y desarrollo de su hijo que por las cuestiones médicas y, sin embargo, muy pocas discuten con su pediatra estas preocupaciones (Hickson et al. 1983). Este sistema de cuestionarios contestados por las familias (cuidadores principales) aporta también como ventaja la inclusión de información de difícil observación directa en el proceso de evaluación (cribado entre otras). Además, los cuidadores (padres/madres) son reforzados como una parte muy importante en todo el proceso, promoviendo así su papel activo en la implementación de estrategias posteriores de intervención.

En cuanto al contenido de los cuestionarios, aunque deben ser simples en sus planteamientos, deberían tener suficientes ítems de cada área del desarrollo (motor, lenguaje comprensivo y expresivo, desarrollo socio-emocional, cognitivo, etc.) como para, en caso de puntuaciones bajas en una de estas áreas, poder orientar al evaluador hacia qué área evaluar con un screening de nivel II. En general la selección de las habilidades a evaluar se establecen siguiendo dos criterios: a) los hitos evolutivos más significativos y b) conductas significativas en la sintomatología de un trastorno. El primer criterio de selección permitirá valorar el nivel de retraso en el desarrollo si es que existe. Mientras que el segundo permitirá el ajuste de la conducta manifiesta sobre unos síntomas potencialmente alarmantes. Una combinación de ambos mejoraría la sensibilidad y la especificidad del instrumento de detección.

Una detección basada en una única prueba no tiene en cuenta la naturaleza dinámica del desarrollo del niño/a. El resultado de un test de desarrollo en un momento determinado en el tiempo puede estar influenciado por diversos factores. El desarrollo es un proceso dinámico no lineal, se caracteriza por rachas, mesetas y hasta regresiones. Una sola instantánea en un punto en el tiempo, no proporciona información sobre la trayectoria de desarrollo del niño/a. Esta reflexión nos lleva a diferenciar entre los test de cribado y los sistemas de vigilancia.

Los tests de detección de nivel I, se aplican o deben aplicarse a toda la población, y su objetivo es distinguir entre aquellas personas que tienen más riesgo de padecer algunos trastornos de aquellas otras de riesgo menor. El sistema de cribado del desarrollo ideal sería aquel que cubriera todas las áreas del desarrollo, en diferentes momentos a lo largo de la primera infancia, formando así un sistema de vigilancia del desarrollo. En este sentido, se podrían y deberían combinar diferentes herramientas tanto de desarrollo general (nivel I) como de patologías específicas (Nivel II) (Drotar et al. 2008).

Teniendo en cuenta la revisión realizada, se observa que la mayoría de los instrumentos de cribado generales encontrados están en inglés, solo algunos de ellos están traducidos al castellano (se entiende que es debido a la presión y/o necesidad de población hispana que reside en Estados Unidos), pero son muy pocos los que están validados en España. Las revisiones o estudios normativos, cuando existen, suelen adolecer de un estudio psicométrico profundo. Sería deseable poder confirmar el porcentaje de respuesta de los ítems cuando estos reflejan hitos evolutivos, con la finalidad de determinar la adecuación del hito a la edad para la que ha sido seleccionado como representante típico. Con respecto al cálculo de la fiabilidad, en general, los resultados manifiestan un nivel medio-alto de fiabilidad. No obstante, se hace notar que la medida de fiabilidad utilizada en la mayoría de las ocasiones hace referencia a la consistencia interna. Este indicador, sin embargo, no garantiza que el instrumento proporcione una medida estable. La fiabilidad como estabilidad de la medida suele medirse mediante el test re-test. Este procedimiento, sin embargo, no es el utilizado en la mayoría de los estudios consultados, posiblemente debido al coste que supone la doble aplicación. Otra medida de fiabilidad ausente que debería tenerse en cuenta en estos casos hace referencia a la consistencia entre jueces. Este indicador se 
hace especialmente relevante en los sistemas y cuestionarios diseñados para que los contesten los/as padres/madres. Un mismo niño/a es observado por varios jueces y sus puntuaciones deberían ser las mismas si las instrucciones y el objeto de observación están bien definidos.

Con respecto a la validez de los sistemas de detección en general se toma como contraste el resultado sobre el mismo individuo de escalas del desarrollo como la Battelle o la Bayley. Siendo adecuado este contraste, no es suficiente sobre todo cuando hablamos de validez de diagnóstico. En este sentido se hace necesario valorar adecuadamente el criterio de contraste. Lo adecuado es conseguir el diagnóstico definitivo, aunque ello obligue en muchos casos a realizar o estudios clínicos o estudios de seguimiento a lo largo del desarrollo del niño/a.

Por último, se deben destacar las ventajas aportadas por las nuevas tecnologías, en particular por la distribución de la información por la red. Existen muchas pruebas con versiones electrónicas o bien que se distribuyen con software de corrección, facilitando la aplicación de los instrumentos. En este sentido, la integración de las facilidades multimedia de la red podrá permitir que las familias con niveles más bajos de comprensión lectora puedan cumplimentar los cuestionarios con menos problemas. A su vez, un sistema electrónico de agenda puede hacer que las familias reciban los avisos oportunos para que cumplimenten los cuestionarios en el momento adecuado según la edad de los/as niños/as.

En definitiva, aunque hay un gran camino ya recorrido y el número de cuestionarios y sistemas de detección es considerable, existe la necesidad en nuestro país de realizar los estudios de validación y normalización necesarios o el desarrollo de sistemas propios. 
AAP (I994): "Committee on Children with Disabilities. Screening infantas and young children for developmental disabilities”. Pediatrics, 93: 863-865.

AAP (200Ia): "Committee on Children with Disabilities. Developmental surveillance and screening of infants an youn children". Pediatrics, г08: 192-196.

AAP (200Ib): Developmental surveillance and screening of infants and young children. Committe on children with disabilities. American Academy of Pediatrics.

AAP (2006): "Identifying Infants and Young Children With Developmental Disorders in the Medical Home: An Algorithm for Developmental Surveillance and Screening”. Pediatrics, I: 405-420.

Aaronson, N. et al. (2002): "Assessing health status and quality-of-life instruments: Attributes and review criteria". Quality of Life Research, I I: 193-202.

Alcantud, F. et al. (2015): Sistema de Detección Precoz de Trastornos del Desarrollo (SDPTD): Construcción, validación y manual de uso (Versión I.o). Madrid: Real Patronato sobre Discapacidad.

Alpern, G. (2007): Developmental Profile. Los Angeles California: Western Psychological Services.

Arruabarrena, I. y de Paul, J. (2OI2): "Early Intervention Programs for Children and Families: Theoretical and EMpirical Bases Supporting their Social And Economic Efficiency". Psychosocial Intervention, 2 (2I): I I 7-I 27 .

Artigas-Pallarés, J. (20I I): “Trastornos del neurodesarrollo: Conceptos básicos, en ArtigasPallarés, J. y Narbona, J.: Trastornos del Neurodesarrollo. Barcelona: Viguera Ediciones.

Aylward, G. (I997): "Conceptual issues in developmental screening and assessment".
Journal of Developmental and Behavioral Pediatrics, I 8: 340-349.

Barnes, K. y Stark, A. (I975): “The Denver Development screening test". American Journal of Public Health: 363-369.

Bayley, N. (2006): Bayley scales of infant and toddler development. 3 rd Edn Screening test Manual. San Antonio Texas: Pearson Education Inc.

Bedford, H. et al. (2013): Measures of child development: A review. Centre for Paediatric Epidemiology and Biostatistics.

Bliss, S. y Newborg, J. (2007): "Battelle Developmental Inventory Sencon Edition”. Journal of Psychoeducational Assessment, 25 (4): 409-4I 5 .

Bradley-Johnson, S. y Johnson, C. (200I): Cognitive abilities scale ( 2 nd ed). Austin Texas: Pro-Ed.

Brigance, A. y Glascoe, F. (2002): Brigance Infant and Toddler Screen, Massachusetts: Hawker Brownlow Education.

Brothers, K. et al. (2008): "PEDS: Developmental Milestones- An Accurate Brief Tool for Surveillance and Screening”. Clinical Pediatrics, 47 (3): 27I-279.

Bryson, S. et al. (2008): "The autism Observation Scale for Infants: Scale development and reliability data". Journal of Autism Developmental Disorders, 38 (4): 73 I-738.

Camp, B. W. (2006): "What the clinician really needs to know: Questioning the clinical usefulness of sensitivity and specificity in studies of screening test". Journal of Developmental and Behavioral Pediatrics, 27: 226-230.

Coonrod, E. y Stone, W. (2005): "Screening for autism in young children", en Volkmar, F. et al.: Handbook of Autism and Pervasive Developmental Disorders. Hoboken NJ: John Wiley \& Sons. 
Cox, A. et al. (I999): “Autism spectrum disorders at 20 and 42 months of age: stability of clinical and ADI-R diagnosis". Journal of Child Psychology ans Psychiatry ans Allied Disciplines, 40 (5): 719-732.

Creighton, D. y Sauve, R. (I988): “The Minnesota Infant Development Inventory in the developmental screening of high-risk infants at 8 months". Journal of Behavior Sciences, 20 : 424-433.

Dawson, S. et al. (2000): "Case study of the development of an infant with autism from birth to two years of age". Journal of Applied Developmental Psychology, 2 I: 299-3 I 3 .

Drotar, D. et al. (2008): Pediatric developmental screening: understanding and selecting instruments. Nueva York: Commonwealth Fund.

Easterbrooks, M. y Goldberg, W. ( I984): “Toddler development in the family: Impact of father involvement and parenting characteristics". Child Development, 55 (3): 740-752.

Erikson, J. (I996): The Infant-toddler developmental assessment (IDA): A familycentered transdisciplinary assessment process. Washington DC: Zero to Three: The National Center for Infants, Toddlers and Families.

Fenichel, G. (I988): Clinical pediatric neurology. Philadelphia: WB Saunders.

Fewell, R. y Langley, M. (I984): DASI-II Developmental activities screening inventory. Austin Texas: Pro-Ed.

Filipek, P. et al. (2000): "Practice parameter: screening and diagnosis of autism". Neurology, $4(55): 468-479$.

Frankenburg, W. y Dodds, J. (I967): “The Denver Developmental Screening Test". The Journal of Pediatrics, 7I (2): I8I-I9I.

Frankenburg, W. et al. (I97I): "Validity of Denver developmental screening test". Child Development: 475-485.

Frankenburg, W. et al. (I992): “The Denver II: a major revision and restandardization of the Denver Developmental Screening Test”. Pediatrics, 89 (I): 9I-97.
GAT, Federación Estatal de Asociaciones de Profesionales de Atención Temprana (2000): Libro Blanco de Atención Temprana. Madrid: Real Patronato sobre Discapacidad.

Glascoe, F. (I997): "Parents' concerns about children's development: pre-screening technique or screening test?". Pediatrics, 99: 522-528.

Glascoe, F. (I998): Parent's Evaluation of Developmental status (PEDS). Nashville: Ellsworth \& Vandermeer.

Glascoe, F. et al. (I992): “Accuracy of the DenverII in developmental screening”. Pediatrics, 89 (6): I22I-I 225 .

Gollenberg, A. et al. (2010): “Concurrent validity of the parent-completes Ages and Stages Questionaires. 2nd Ed. with the Bayley Scales of INfant Development II in low-risk sample”. Child: Care, Health \& Development, 36 (4): 485-490.

Gracy, M. et al. (2010): Developmental and SocialEmotional Screening Instruments for Use in Pediatric Primary Care in Infants and Young Children. Nueva York: Children's Health Fundation.

Greenwood, C. et al. (2005): "Individual growth and development indicators (IGDIs): Tools for assessing intervention results for infants and toddlers". Focus on behavior analysis in education: Achievements, challenges, and opportunities: I03-I 24 .

Halle, T. et al. (20I I): Understanding and choosing Assessments and Developmental Screeners for Young Children Ages 3-5: Profiles of Selected Measures, Washington, DC: Office of Planning, Research, and Evaluation, Administration for CHildren and Families, U.S. Departament of Health and Human Services.

Hamilton, S. (2006): "Screening for developmental delay: reliable, easy-to-use tools". Journal of Family Practice, 55 (5): 4I 5-422.

Hardin, B. y Peisner, E. (200I): The Early Learning Accomplishment Profile (E-LAP). Examiner's manual and Reliability and Validity, Chapel Hill: Training Ourreach Project Inc Kaplan Early Learning Company. 
Hickson, G. et al. (1983): “Concerns of mothers seeking care in private pediatric offices: Opportunities for expanding services".

Pediatrics, 72: 619-624.

Hix-Small, H. et al. (2006): "Impact of implementing Developmental Screening at I 2 and 24 months in a Pediatric Practice". Pediatrics, 2 (I 20): 38 I-388.

Hresko, W. et al. (1994): Developmental Observation Checklist System. Austin Texas: Pro-Ed.

Ireton, H. (I992): Child Development Inventory Manual. Minneapolis: Behavior Science Systems.

Ireton, H. (2004): Child Development Review Manual. Minneapolis: Behavior Science Systems.

Ireton, H. y Glascoe, F. (I995): “Assessin Children's Development Using Parent's Reports: The Child Developmen Inventory". Journal of Clinical Pediatrics, 34 (5): 248-255.

Ireton, H. et al. (I977): “Minnesota Child Development Inventory: Identification of children with developmental disorders". Journal of Pediatric Psychology, 2 (I): I 8-22.

Johnson, C. y Bradley-Johnson, S. (2002): "Construct stability of the cognitive abilities scale for infants and toldders". Journal of Psychoeducational Assessment, 20 (2): I44I 5 I.

Johnson, C. y Myers, S. (2007): "Identification and evaluation of children with autism spectrum disorders". Pediatrics, 5 ( ( 20 ).

Johnson, S. y Marlow, N. (2006): "Developmental screen or developmental testing?”. Early Human Development, 82: I73-183.

Kerstjems, J. et al. (2009): "Support for the global feasibility of the Ages and Stages Questionaire as developmental screener". Early human development, 85 (7): 443-447.

Kleinman, J. et al. (2008): "Diagnostic stability in very young children with Autism Spectrum Disorders". Journal of Autism And Developmental Disorders, 38: 606-6I 5 .
Kube, D. et al. (I988): "Classification of Developmental Delays”. Seminars in Pediatric Neurology, 5 (I): 2-I 4 .

Leiarraga, H. et al. (2005): Prueba Nacional de Pesquisa, PRUNAPE, Manual Técnico, Buenos Aires: Fundación Hospital Garran.

Lord, C. y Corsello, C. (2005): "Diagnostic instruments in autistic spectrum disorders", en Volkmar, F. et al.: Handbook of Autism and Pervasive Developmental Disorders. Hoboken NJ: John Wikey \& Sons.

Meisels, J. y Provence, S. (1989): Guidelines for identifying young disabled and developmentally vulnerable children and ther families.

Washington DC: Bational Center for Clinical Infant Programs.

Miler, L. (I993): FirstSTEP: Screening Test for Evaluating Preschoolers. Manual. San Antonio Texas: Pearson.

Moodie, S. et al. (20I4): Early childhood developmental screening: A compendium of measures for children ages birth to five. Washington: Office of Planning, Research and Evaluation, Administration for Children and Families. US Departament of Health and Human Services.

Muñoz, J. et al. (2006): "Autismo, Identificación e intervención temprana”. Acta de Neurologia Colombiana, 2 (22).

Newborg, J. (2005): Battelle Developmental Inventory. 2nd Edition: Examiner's manual, Itasca IL: Riverside.

Pomés, P. (2012): Examination of the spanish translation of developmental screening instrument, Oregon: University of Oregon.

Provence, S. y Apfel, N. (200I): Ifant-Toddler and Family Instrument (ITFI), Paul H Brookes.

Reuter, J. y Gruber, C. (2000): Kent Inventory of Developmental Skills. Kent, Ohio: Western Psychological Services.

Reuter, J. et al. (1996): Kent Inventory of Developmental Skills (KIDS), Los Angeles: Western Psychological Services.

Ringwalt, S. (2008): Developmental screening and assessment instruments with an emphasis 
on social and emotional development for young children ages birth through five, Chapel Hill: The University of North: FPG Child Development Institute The National Early Childhood Technical Assistance Center.

Roxswell, S. (I993): FirstSPET: Screening test for evaluating preschoolers, Harcourt Brace Javanovich: Psychological Corporation.

Rydz, D. et al. (2006): "Screening for Developmental Delay in the Setting of a Community Pediatric Clinic: A Prospective Assessment of Parent-Report Questionnaires”. Pediatrics, 4 (I I 8).

Sheehan, R. (I988): "Involvement of parents in early childhood assessment", en Wachs T. y Sheehan, R. (Eds): Assessment of young developmentally disabled children, Nueva York: 75-90.

Shonkoff, J. et al. (I979): "Primary care approaches to developmental disabilities”. Pediatrics, 4 (64): 506-5I4.
Squires, J. et al. (I997): "Revision of a ParentCompleted Developmental Screening Tool: Ages and Stages Questionaires". Journal of Pediatric Psychology, 22 (3): 3I3-328.

Squires, J. et al. (I998): "Parent-completed Developmental questionnarires: Effectiveness with low and middke income parents". Early Childhood Research Quaterly: 345-353.

Terwee, C. et al. (2007): “Quality criteria were proposed for measurement properties of health status questionnaires". Journal of Clinical Epidemiology, 60: 34-42.

Vericat, A. y Orden, A. B. (20I0): “Herramientas de Screening del Desarrollo Psicomotor en Latinoamérica". Revista chilena de pediatría, $8 I(5): 39$ I-40I.

Voress, J. y Maddox, T. (1998): Developmental assessment of young children (DAYC-2Ed). Austin Texas: Pro-Ed. 